\title{
¿QUÉ LE VENDEMOS, REINA? EL USO DE LOS VOCATIVOS EN LA FERIA DEL AGRICULTOR
}

\author{
Leyla Hasbún Hasbún
}

\begin{abstract}
RESUMEN
El presente estudio investiga el uso no recíproco de vocativos cariñosos entre extraños en lugares públicos. Se grabaron un total de 99 transacciones en una Feria del Agricultor en San José para determinar su uso y frecuencia. Los resultados indican que, en cerca de la mitad de los diálogos, no se usó vocativo alguno. En la otra mitad, hubo aproximadamente el mismo número de diálogos en los que se usaron vocativos de respeto o deferencia que de diálogos donde se utilizaron vocativos cariñosos. El estudio también discute posibles motivos subyacentes al uso de vocativos cariñosos.
\end{abstract}

\begin{abstract}
This study investigates the nonreciprocal use of terms of endearment between strangers in a public place. A total of 99 transactions were recorded at a Farmers' Market in San José to determine use and frequency. Results indicate that in about half of the dialogues, there was no overt form of address. In the other half, $41.5 \%$ of the dialogues included traditional forms of respect while in $58.5 \%$ terms of endearment were used. This study also speculates about the underlying motives for the use of terms of endearment.
\end{abstract}

\section{Introducción}

Para tener éxito, la comunicación entre las personas debe regirse por las reglas de cortesía imperantes en la comunidad lingüística donde se desenvuelven los participantes. Brown y Levinson (1978: 66) han propuesto una teoría de la cortesía que ellos consideran universal, cuyo objetivo es analizar las relaciones humanas. En dicha teoría, se parte del supuesto de que las personas establecen relaciones con el fin de satisfacer una gran variedad de necesidades. En el campo específico de la cortesía, los autores argumentan que las personas tienen la necesidad de preservar su dignidad o su imagen social ("face"). Ellos describen esta imagen social como "algo que se invierte en el campo emocional, y que puede ser perdido, mantenido o realzado y a lo que debemos prestar atención constantemente durante una interacción" (Traducción de la autora).

Brown y Levinson sostienen que hay dos tipos de imagen social: una negativa y otra positiva. La negativa es el derecho a la libertad de acción y a no tener que tolerar abusos o imposiciones de los demás. Existen muchas estrategias de cortesía que se emplean para evitar 
menoscabar estas necesidades de nuestros semejantes. Son formas de mostrar respeto, de evitar ocasionar molestia a los demás y de evidenciar consideración para con sus sentimientos y susceptibilidades. Se manifiesta este tipo de cortesía, por ejemplo, cuando las personas se esfuerzan por expresarse apropiadamente en términos de distancia social. Conscientemente, se escogen aquellas formas de tratamiento que reflejen un grado adecuado de respeto hacia el interlocutor o que marquen las diferencias de estatus existentes. Igualmente, las personas se excusan o utilizan alguna forma cortés para mitigar la imposición cuando se tiene que pedir un favor con el cual se puede incomodar al interlocutor.

Por otro lado, la imagen social positiva se relaciona con la imagen que se tiene de sí mismo y con la necesidad de recibir la aprobación de al menos algunos miembros de la comunidad, es decir, de ser valorados y aceptados por ellos. La cortesía positiva está más orientada hacia la solidaridad. Por esta razón, por ejemplo, se saluda o se muestra preocupación por los problemas de otros. Con este comportamiento se manifiesta el deseo de destacar las actitudes y valores que se comparten con otros. La cortesía es, en resumen, el deseo de preservar ambos tipos de imagen social ya que, en el diario vivir, muchas acciones, propias y de otros, pueden ser percibidas como amenazas a esta imagen.

\section{La cortesía verbal y la elección de vocativos}

El uso que hagamos de la lengua, por ejemplo, la escogencia del vocabulario que empleamos, puede violentar las normas de cortesía y, por ende, ofender a la persona con quien hablamos. Esto puede ocurrir cuando no tomamos en cuenta los sentimientos de nuestros interlocutores o no hacemos un esfuerzo por utilizar estrategias, como las descritas anteriormente, las cuales permiten minimizar el riesgo de amenazar la imagen social del interlocutor. Podemos ofender a una persona tanto al tratarla con demasiada confianza o familiaridad como cuando la tratamos en forma excesivamente distante. En estos casos, la cortesía consiste en seleccionar la expresión lingüística oportuna, de acuerdo con la circunstancia social que enfrentamos, pero desde el punto de vista del interlocutor y no necesariamente del de la persona que habla.

Un área potencialmente conflictiva es el uso de vocativos cariñosos que comunican excesiva confianza entre personas extrañas. Se entiende que ciertas palabras de afecto están reservadas para aquellos con los que tenemos una relación cercana o íntima. Si las recibimos de ellos, se refuerza nuestra auto imagen y nos sentimos valorados y aceptados. Sin embargo, si vienen de alguien que no conocemos, muy especialmente si una mujer las recibe de un hombre, su imagen social podría verse amenazada. Muchas personas pueden interpretar este acto como una violación a su derecho al respeto, como una imposición y, en casos extremos, como acoso.

Esta percepción es la que probablemente indujo a una ciudadana costarricense a enviar la siguiente carta a un periódico local:

\footnotetext{
"Negra", "Mi vida", "Papito..." es la forma en que tratan a los clientes los vendedores de jocotes, de lotería, de tiendas, etc. Igual hacen las telefonistas de empresas privadas u oficinas del Gobierno. ¿Por qué no contratar personas educadas, amables (pero no tanto)? Quien atiende al público es la cara del negocio o la institución, pero en lugar de dar una buena impresión, ofenden (La Nación, 22 de agosto de 1999: 13-A).
} 
Evidentemente, el tema de una conversación, los roles de los participantes, el lugar y los propósitos de la comunicación determinan la escogencia apropiada de nuestras palabras. La queja de esta ciudadana refleja la incompatibilidad que ella percibe entre los vocativos utilizados por una variedad de personas que atienden al público y la circunstancia social en que se encuentran. Curiosamente, existe la posibilidad de que un vendedor de jocotes, por ejemplo, emplee el vocativo "mi vida" como una estrategia de cortesía, como un halago, con la esperanza de que le permita reforzar la imagen positiva de una cliente potencial. Sin embargo, su cliente puede interpretar el mismo hecho como una trasgresión a su derecho a no tener que tolerar abusos de confianza o imposiciones de otros, lo que interpreta como una falta de respeto.

El objetivo principal del presente estudio es determinar qué tipos de vocativos reciben las mujeres en un sitio público como la Feria del Agricultor en la ciudad de San José, donde se establece una relación entre extraños marcada, además, por la diferencia de estatus social. El segundo objetivo es especular sobre los motivos que puedan tener los hablantes a la hora de escoger esos vocativos. Partimos de la premisa de que estos propósitos pueden ser neutros como cuando no se usa vocativo alguno, puede ser el respeto como cuando se elige "doña", o puede ser el deseo de expresar solidaridad, o tal vez poder, cuando se recurre al uso de "mi amor". En este último caso, el receptor del vocativo puede aceptarlo ya sea como una manifestación de o como una violación a las normas de cortesía.

\section{Algunos estudios sobre el uso de vocativos realizados en los Estados Unidos, Inglaterra y Australia}

Nancy Henly (1977) argumenta que muchas de las características de las interacciones que se suscitan entre hombres y mujeres, las cuales tradicionalmente han sido interpretadas como evidencia de solidaridad, en realidad son consecuencia de la dominación masculina. En su opinión, tanto el lenguaje verbal como el no verbal utilizados por las personas que están en una posición de poder, por lo general hombres, tienen el propósito de mantener una jerarquía social existente.

En su libro Language and the woman's place, Robin Lakoff (1975: 80) argumenta que, en la sociedad norteamericana, el uso de vocativos cariñosos tales como "dear" y "honey" entre personas que no tienen una relación cercana no es recíproco. Añade que aunque las personas de ambos sexos las utilizan, esto sucede bajo condiciones diferentes. Para esta autora, muchas mujeres en una situación de subordinación social, como sucede con las meseras o vendedoras de tiendas, las usan para dirigirse tanto a hombres como a mujeres. Los hombres heterosexuales, por el contrario, nunca las utilizan para dirigirse a otros hombres, y cuando las usan con una mujer con la que no tienen una relación sentimental o de amistad, la mujer está invariablemente en una posición de inferioridad.

Wolfson y Manes (1980) encontraron asimetría en situaciones de servicio donde los dependientes y las dependientes de tiendas usaban palabras cariñosas para dirigirse a las mujeres, pero que nunca lo hacían con los clientes masculinos.

En sus estudios en Australia, Poynton (1985: 80-2) opina que, dado que una vasta mayoría de las mujeres tiene trabajos de bajo estatus y, por lo general, sus jefes son hombres, el uso de vocativos cariñosos en forma no recíproca no la sorprende. Comenta que lo más interesante 
de esta asimetría es su naturaleza ya que pareciera que es socialmente aceptado que a las mujeres, al igual que a los niños, se les digan en público palabras cariñosas que, tradicionalmente, están reservadas para situaciones de intimidad. No sucede lo mismo con los hombres. Finalmente, comenta que en Australia es común que hombres de estatus social diferente, como podría ser la relación que se establece entre cliente y empleado en una estación de gasolina o en un bar, usen entre sí el término "mate" el cual no tiene connotación de intimidad sino más bien de solidaridad.

Wolfson (1989) aduce que sus investigaciones etnográficas indican que "el hecho de que para referirse a las mujeres se usen en público términos cariñosos en forma no recíproca, sin importar la edad o el estatus de éstas, puede ser considerado como un indicio de que las mujeres son generalmente tratadas con menos respeto que los hombres en la sociedad estadounidense" (Traducción de la autora). Opiniones similares expresa McConnell-Ginet (1978).

Por otro lado, Holmes (1992: 300) asegura que en el norte de Inglaterra muchos vendedores de periódicos, choferes de autobús y vendedores de pasajes de tren usan el término "love" para referirse a todas las personas sin importar qué tan bien las conocen y, con frecuencia, sin distinción de género. Este hallazgo es de naturaleza diferente a los anteriores ya que no refuerza la existencia de tratos diferentes para las personas dependiendo de su género.

En un estudio posterior, Holmes (1998) opina que los cumplidos, al igual que las palabras cariñosas utilizadas por extraños:

\footnotetext{
Toman fuerza del contexto en que se lleva a cabo la interacción entre las personas. Cuando se usan en forma no recíproca por parte de los superiores a sus subalternos, este tratamiento puede reflejar una relación subyacente de poder social, la cual coloca a las mujeres en una posición claramente subordinada a los hombres. Por otro lado, cuando [los cumplidos y las palabras cariñosas] se usan entre iguales o entre amigos, especialmente cuando se trata de mujeres de igual condición social y que además son amigas, pueden considerarse la quintaesencia de una estrategia positiva de cortesía (Traducción de la autora).
}

Finalmente, se ha llevado a cabo otro tipo de investigaciones que tiene que ver con violaciones a lo que llaman "civilidad pública" en encuentros entre extraños en lugares públicos. Por ejemplo, Gardner $(1980,1995)$ en sus estudios que analizan la relación que existe entre algunas variables tales como el género, el grupo étnico, la orientación sexual y la discapacidad y diferentes manifestaciones de acoso, concluye que este acoso ejerce un poderoso control sobre el bienestar de las mujeres. Sin embargo, los hombres y las mujeres reaccionan a este fenómeno y lo interpretan en formas muy diferentes.

\section{Estudios sobre la cortesía verbal realizados en Costa Rica}

A pesar de que se han escrito numerosos artículos sociolingüísticos en Costa Rica (por ejemplo, Hasbún y Solís, 1997, 1999; Hernández, 1999), el estudio de la cortesía verbal en Costa Rica aún está en ciernes. Arias (2002) realizó una investigación socio-pragmática del uso de tratamientos en el área metropolitana de San José. Diseñó una encuesta y recogió datos de 30 personas. Aquellos resultados de su tesis que directamente se relacionan con el presente estudio, es decir, situaciones donde un desconocido presta un servicio que lo coloca en una posición de menor estatus social, se resumen en los tres siguientes cuadros. En ellos, la primera columna menciona al posible interlocutor de los encuestados, la segunda incluye los vocativos que los varones encuestados dicen usar cuando interactúan con la persona mencionada en la primera columna, y la tercera columna presenta los vocativos reportados por las mujeres. 
Cuadro 1: Uso de vocativos en la primera generación: hablantes de 20 a 34 años

\begin{tabular}{|c|c|c|}
\hline Interlocutor & Vocativos empleados por hombres & Vocativos empleados por mujeres \\
\hline Chofer de bus & señor, jefe & señor \\
\hline Vendedor de tienda & señor, muchacho, joven, jefe & muchacho, joven, señor \\
\hline Vendedora de tienda & $\begin{array}{l}\text { muchacha, señorita, señora, } \\
\text { joven, linda }\end{array}$ & $\begin{array}{l}\text { muchacha, señorita, señora, } \\
\text { joven }\end{array}$ \\
\hline Mesero & $\begin{array}{l}\text { señor, joven, mesero, muchacho } \\
\text { jefe, bróder, mae }\end{array}$ & joven, señor, muchacho \\
\hline Mesera & $\begin{array}{l}\text { señorita, señora, mesera, muchacha, } \\
\text { joven, reina o reinita }\end{array}$ & señorita, señora, muchacha, joven \\
\hline
\end{tabular}

En el Cuadro 1, llama la atención el hecho de que ninguna mujer reportó el uso de vocativos cariñosos ni para hombres ni para mujeres. Por el contrario, los hombres utilizaron términos solidarios tales como "jefe" o "bróder" exclusivamente para dirigirse a otros hombres y emplearon tratamientos que reflejan mayor intimidad como "linda" para dirigirse a una vendedora de tienda y "reina" para una mesera.

En la segunda generación, es decir, hablantes de 35 a 54 años, hay mayor variedad.

Cuadro 2: Uso de vocativos en la segunda generación: hablantes de 35 a 54 años

\begin{tabular}{|l|l|l|}
\hline Interlocutor & Vocativos empleados por hombres & Vocativos empleados por mujeres \\
\hline Chofer de bus & señor & señor, ñatico \\
\hline Vendedor de tienda & joven, jefe, señor, muchacho, primo & $\begin{array}{l}\text { muchacho, joven, señor, caballero } \\
\text { corazón, nato, muñeco, mi amor }\end{array}$ \\
\hline Vendedora de tienda & $\begin{array}{l}\text { señorita, negra, negrita, muchacha } \\
\text { reina, reinita }\end{array}$ & $\begin{array}{l}\text { señorita, muchacha, señora } \\
\text { joven, reina, reinita }\end{array}$ \\
\hline Mesero & joven, señor, amigo & $\begin{array}{l}\text { señor, joven, caballero, guapo, corazón, } \\
\text { nato, mi amor }\end{array}$ \\
\hline Mesera & señorita, señora, joven & $\begin{array}{l}\text { señorita, señora, joven, reina, reinita, } \\
\text { ñatica, macha }\end{array}$ \\
\hline
\end{tabular}

Nuevamente, los hombres utilizan términos solidarios para hablar con personas de su mismo género y términos cariñosos para las mujeres. A diferencia del grupo anterior, estos varones reportan el uso de vocativos cariñosos únicamente para vendedoras de tienda y no para 
meseras. Sin embargo, a diferencia del primer grupo, las mujeres también usaron vocativos cariñosos para los hombres, algunos de los cuales, como "ñato" o "ñatico", sería importante investigar, ya que parecieran no ser muy frecuentes en el habla costarricense.

En la tercera generación se reportó otro cambio interesante.

Cuadro 3: Uso de vocativos en la tercera generación: hablantes de más de 55 años

\begin{tabular}{|l|l|l|}
\hline \hline Interlocutor & Vocativos usados por hombres & Vocativos usados por mujeres \\
\hline Chofer de bus & don, señor & señor, joven \\
\hline Vendedor de tienda & don, muchacho, pito & joven, señor, muchacho \\
\hline Vendedora de tienda & joven, señorita, señora, muchacha & $\begin{array}{l}\text { muchacha, señorita, joven, señora } \\
\text { mamita }\end{array}$ \\
\hline Mesero & joven, señor & joven, señor \\
\hline Mesera & $\begin{array}{l}\text { joven, señora, señorita, chiquilla, } \\
\text { chiquita, mi amor }\end{array}$ & señorita, señora, joven \\
\hline
\end{tabular}

Comparados con los sujetos de los otros dos grupos, los hombres de mayor edad utilizan menos términos que reflejan solidaridad para las personas de su mismo género. El único reportado es "pito". Para las mujeres, mencionaron algunos vocativos cariñosos que acentúan la diferencia de edad entre los interlocutores, como el caso de "chiquilla". En cuanto a las mujeres, ellas tampoco reportaron vocativos cariñosos para los hombres. En cuanto a su trato con otras mujeres, solamente mencionaron "mamita", otro vocativo que puede utilizarse para enfatizar la brecha generacional.

Se debe destacar que, en su trabajo, Arias considera que todos los vocativos cariñosos reportados son manifestaciones de cortesía (ver p. 52 y p. 53). Explica que son formas de congraciarse con personas del sexo opuesto. Esta interpretación de los datos es muy diferente a la de la psicóloga Henley (1977) o la educadora Poynton (1985), por ejemplo.

También con relación al estudio de Arias, en una investigación futura, sería importante preguntarle a los sujetos entrevistados qué vocativos reciben ellos de los interlocutores mencionados en la encuesta. Esto nos daría una visión más global de estas transacciones.

Por su parte, Pavez (2002) realizó un estudio sobre la cortesía conversacional y la imagen social en las salas de chat frecuentadas por costarricenses. A pesar de que el contexto donde se realizó el estudio de Pavez es muy específico, su descripción del medio, es decir, las salas de chat, es relevante:

Es bastante hostil para las mujeres, pues tienen menos opciones en las cuales ser encasilladas. Generalmente se las asume como interesadas en, y necesitadas de, relaciones íntimas y nada más. Además, querrían que las hagan sentir bien apelando a su físico y refiriéndose a ellas con cariño (164). 
Esta aseveración da una interesante interpretación, desde el punto de vista de una mujer, de cómo son percibidas las mujeres por sus interlocutores en las salas de chat y cómo esta percepción se manifiesta en el lenguaje escogido por los hombres.

En un estudio sobre la cortesía verbal en Costa Rica, Murillo (2003) insiste en la importancia de utilizar conceptos de imagen social y de cortesía verbal flexibles que permitan entender verdaderamente las complejidades socioculturales de una comunidad lingüística. En cuanto al uso de vocativos, tema central de la presente investigación, afirma lo siguiente:

\footnotetext{
Los apelativos, usados deícticamente, constituyen verdaderos actos de habla cuya función primordial es hacer evidente una relación social entre hablante y oyente. Además, codifican información relevante para el análisis de la cortesía verbal, pues se convierten en estrategias que los hablantes usamos para lograr ciertos propósitos (138).
}

Una mejor comprensión de estos propósitos que menciona Murillo nos permitirá describir con mayor objetividad las complejidades de las relaciones entre las personas.

\section{Metodología}

Los datos utilizados en esta investigación fueron recogidos mediante grabaciones realizadas en la Feria del Agricultor ubicada en Plaza González Víquez, en la ciudad de San José. Durante tres sábados consecutivos, la investigadora, mujer de mediana edad, realizó sus compras en dicho mercado en compañía de su esposo y de sus dos hijos universitarios. Utilizando una grabadora Sony TCM-S67V grabó todas las interacciones que llevó a cabo con los vendedores de frutas y verduras. No se hizo ningún esfuerzo por ocultar la grabadora. Al finalizar este período, se transcribieron tres cintas de audio de 60 minutos de duración cada una y se identificó el género de los participantes. A continuación, un diálogo ilustrativo:

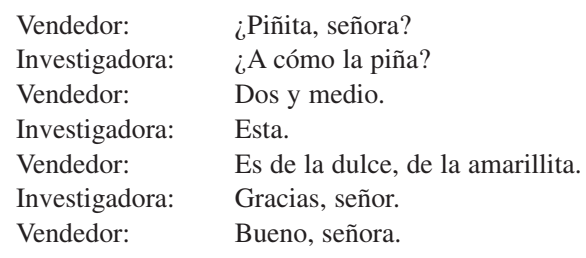

El segundo paso consistió en el procesamiento y análisis de los datos. Para ello, se revisaron uno a uno los diálogos registrados, se preparó una lista de los vocativos empleados por los vendedores y las vendedoras y se determinó el número de diálogos en los que no se utilizó ninguno. Al inicio de este proyecto, se planeó hacer una división de la muestra por género del vendedor. Sin embargo, debido a las características particulares de esta población de vendedores, este análisis resultó inapropiado, ya que 93 de los diálogos se llevaron a cabo con vendedores y únicamente 6 con vendedoras. En vista de que la muestra de vendedoras es tan pequeña, y que, por lo tanto, el uso de vocativos por parte de un único sujeto puede fácilmente sesgar los resultados, no fue posible inferir si el comportamiento lingüístico de los vendedores y las vendedoras es significativamente diferente. 
Antes de iniciar el análisis y la discusión de la información obtenida, es conveniente mencionar los datos que fue necesario desechar. En primer lugar, por razones obvias, se excluyeron de la muestra dos diálogos en los que el vendedor interactuó exclusivamente con el esposo de la investigadora. En segundo lugar, igual decisión se tomó con otro donde la vendedora afirmó no ser costarricense. Se tomó esta determinación ya que esta persona pertenece a una comunidad lingüística cuyas normas de cortesía verbal pueden ser diferentes a las que prevalecen en Costa Rica. Sin embargo, se procede a transcribir el diálogo ya que tiene valor como elemento de comparación.

$\begin{array}{ll}\text { Vendedora: } & \text { A ver el pinolillo, amor. } \\ \text { Investigadora: } & \text { ¿Qué es lo que tiene el pinolillo? } \\ \text { Vendedora: } & \text { Es legítimo, amor. Yo soy nicaragüense y yo lo hago. } \\ \text { Investigadora: } & \text { ¿Pero qué es lo que lleva? } \\ \text { Vendedora: } & \text { Cacao y maíz tostado. } \\ \text { Investigadora: } & \text { Cacao y maíz tostado. } \\ \text { Vendedora: } & \text { Sí. Y canelita, amor. } \\ \text { Investigadora: } & \text { Y ¿a cómo es? } \\ \text { Vendedora: } & \text { A trescientos, amor. Pero es el legítimo. } \\ \text { Investigadora: } & \text { Y ¿con qué lo bate, con agua o con leche? } \\ \text { Vendedora: } & \text { Con agua, como usted quiera lo bate, y le echa hielo, azúcar y ya se } \\ & \text { lo bebe. Es fresquito de ahora, amor. Lo acabo de ir a moler. } \\ \text { Investigadora: } & \text { Vamos a probarlo. }\end{array}$

La vendedora del ejemplo anterior utilizó cinco vocativos, el mayor número de todos los vendedores, hombres y mujeres. Interesantemente, el vocativo fue siempre el mismo. Esta alta frecuencia de uso conjugado con la falta de variedad parece darle a este comportamiento carácter de muletilla.

\section{Análisis}

Se recogieron un total de 99 diálogos: 29 la primera vez, 34 la segunda y 36 la tercera. De los 99 diálogos, 52 no incluyeron el uso de vocativos mientras 47 sí. De esos 47, en 41 de ellos, se empleó un solo vocativo, mientras que en los 6 restantes se usaron 2 vocativos en cada uno.

En el cuadro 4, se muestra un listado de los vocativos utilizados por los vendedores así como su distribución porcentual. Recuérdese que se había determinado que en 52 de los 99 diálogos $(52.5 \%)$ los sujetos no utilizaron vocativos; por lo tanto, la frecuencia de uso se refiere exclusivamente a los $47(47.5 \%)$ diálogos restantes.

En este cuadro se aprecian algunas situaciones interesantes. En primer lugar, se usaron cuatro vocativos diferentes que pueden considerarse de respeto o de distancia. En conjunto, se usaron 22 veces, lo que representa un $41.5 \%$ del total. Por otro lado, se usaron 12 vocativos diferentes que pueden considerarse de naturaleza íntima o cariñosa, los cuales se usaron, en conjunto, 31 veces, lo que representa un $58.5 \%$ del total. 
Cuadro 4: Vocativos empleados por los vendedores y frecuencia de su uso

\begin{tabular}{|l|c|c|c|}
\hline & \multicolumn{2}{|c|}{ Frecuencia de uso } & \\
\hline Vocativo usado & Absoluta & Relativa & \\
\hline Señora & 13 & $24.5 \%$ & $41.5 \%$ \\
Doña & 6 & $11.3 \%$ & \\
Doñita & 2 & $3.9 \%$ & \\
Señorita & 1 & $1.8 \%$ & $58.5 \%$ \\
\hline Mamita & 3 & $5.7 \%$ & \\
Madre & 1 & $1.8 \%$ & \\
Reina & 12 & $22.7 \%$ & \\
Mi reina & 2 & $3.9 \%$ & \\
Reinita & 1 & $1.8 \%$ & \\
Negra & 3 & $5.7 \%$ & \\
Negrita & 2 & $3.9 \%$ & \\
Amor & 3 & $5.7 \%$ & \\
Mi amor & 1 & $1.8 \%$ & \\
Angelito & 1 & $1.8 \%$ & \\
Linda & 1 & $1.8 \%$ & \\
Amigos* & 1 & $1.8 \%$ & \\
\hline Total & 53 & $100 \%$ & \\
\hline *Este vocativo se refería al grupo familiar. & & \\
\hline
\end{tabular}

En el grupo de los vocativos de distancia, el que se usó con mayor frecuencia fue "señora", con 13 veces que representan un $59.1 \%$ del uso dentro del grupo y un $24.3 \%$ del total de la muestra. En segundo lugar, está “doña"con una frecuencia de uso de 6 veces, que representa $27.3 \%$ del grupo y un $11.3 \%$ del total de la muestra. Estos dos vocativos son probablemente los más usados en el medio costarricense para dirigirse en forma respetuosa a las mujeres que reúnen ciertas características de edad y, en algunos casos, estado civil, especialmente cuando no hay una relación de mucha confianza. Ambos vocativos pueden considerarse equivalentes, aunque probablemente existan preferencias de uso determinadas por variables de índole socioeconómicas como el lugar de procedencia del hablante.

En el grupo de los vocativos cariñosos existe mayor variedad ya que se hallaron 12 en total. El que se usó con mayor frecuencia fue "reina" con 12 veces, lo que representa $38.7 \%$ del uso dentro del grupo y un $22.7 \%$ del total de la muestra. Si a esto se le suma el uso de las variantes "mi reina" y "reinita", los porcentajes de uso se elevan a $48.4 \%$ dentro del grupo y a $28.4 \%$ de la muestra. En segundo lugar, aparece el vocativo "negra" con 3 veces y "negrita" con 2. Estos vocativos representan el $9.6 \%$ del uso dentro del grupo y $16.1 \%$ del total de la muestra. Posiblemente exista una diferencia importante entre la percepción del significado de "reina" y de "negra". El uso del primero es más fácilmente interpretado como una estrategia de cortesía positiva. Si la clienta es una reina, se le puede estar atribuyendo características que, en sentido figurado, la hacen sobresalir entre las demás. No obstante, negra es claramente un vocativo más íntimo y, por consiguiente, más difícil de interpretar como una estrategia de cortesía. 


\section{Discusión}

Los datos indican que en las transacciones comerciales investigadas se dan tres tipos de tratamiento para una compradora de mediana edad: la ausencia de vocativo, el uso de vocativos de deferencia y el uso de vocativos que tradicionalmente han sido asociados con relaciones de intimidad. Las dos primeras situaciones son las esperadas. No obstante, se dio con frecuencia el tercer caso a pesar de que estas interacciones se efectuaron entre extraños y en ellas al cliente se le asigna automáticamente un estatus social más alto. Debido a esta distancia social, se esperaría siempre el uso de vocativos de respeto o deferencia pero, en su lugar, encontramos con cierta frecuencia "angelito" o "negrita".

Como se mencionó, caben diversas interpretaciones para este fenómeno. Retomando el trabajo de Brown y Levinson (1978), podemos pensar que nos encontramos ante el uso de una estrategia de cortesía positiva. Paradójicamente, también podemos considerar que es un abuso o imposición, lo cual constituye una descortesía.

Podemos partir de la hipótesis de que un vendedor de la Feria del Agricultor desea congraciarse con sus clientes. Cuando son mujeres, las trata de halagar mediante el uso de vocativos cariñosos que las hagan sentir aceptadas, es decir, que realcen su imagen social positiva. Supuestamente, esta vinculación hará que la transacción sea exitosa: que la cliente compre sus frutas y verduras porque se siente bien. Igualmente, podemos plantear la hipótesis que el vendedor utiliza vocativos cariñosos como muletilla y que no hay un motivo ulterior, no hay propósito definido.

La cliente, por su parte, puede aceptar los vocativos cariñosos como un cumplido y sentirse complacida o puede ni siquiera percatarse de ellos. En este segundo caso, no les pone atención porque los percibe como palabras huecas, muletillas sin sentido. Sin embargo, también puede sentirse incómoda u ofendida como en el caso de la persona antes referida quien escribió una carta pública a un periódico con el fin de mostrar su desaprobación de esta práctica lingüística. Las personas que reaccionan de esta manera pueden pensar que el origen de este comportamiento es la falta de educación formal, de roce social o de "savoir faire". Otra interpretación es adscribirle a este comportamiento la pérdida de valores tales como el respeto mutuo. Finalmente, podemos estar de acuerdo con Henley (1977), quien considera que se trata de una forma de dominación masculina subyacente o con Wolfson (1989), quien teoriza que es el producto de una sociedad donde se tiende a tratar a la mujer con menor respeto.

Sin embargo, esta última opción presenta un problema de interpretación. Es generalmente aceptado que el uso de la lengua para perpetuar la dominación masculina tiende a darse con mayor frecuencia en situaciones en las que la mujer está sujeta a una relación en la que ella tiene un estatus social inferior. Esto no es precisamente lo que sucede en la Feria del Agricultor. Otra variable que se debe tomar en cuenta es que todas las transacciones registradas se llevaron a cabo en presencia del esposo y los hijos de la investigadora, por lo que es menos probable que los propósitos de los vendedores hayan sido faltar al respeto a la cliente. Además, si consideramos el siguiente ejemplo, podemos ver cómo un mismo vendedor usa vocativos de respeto y de intimidad en una misma conversación.

$\begin{array}{ll}\text { Vendedor: } & \text { ¿Tomatito maduro, señora? } \\ \text { Investigadora: } & \text { Gracias. Péseme a ver cuánto es esto. } \\ \text { Vendedor: } & \text { Kilo y medio. } \\ \text { Investigadora: } & \text { ¿Cuánto es? } \\ \text { Vendedor: } & \text { Trescientos, amor. }\end{array}$


Esta conversación se inicia con el uso de "señora" y termina con el de "amor". Evidentemente, este intercambio de palabras es tan corto que no pareciera suficiente para establecer ningún tipo de lazos afectivos entre los interlocutores, por lo que podríamos concluir que los vocativos más bien aparecen en variación libre.

A pesar de todo lo anterior, me siento muy incómoda cada vez que un extraño utiliza vocativos cariñosos para dirigirse a mí. Por ejemplo, pienso que los vendedores de la Feria del Agricultor que los usaron no respetaron mi derecho a un trato cordial pero digno. No tomaron en cuenta que los traté con respeto. Por lo tanto, para poder entender mejor la motivación subyacente en el uso de vocativos cariñosos entre extraños, me propongo completar la presente investigación con otro proyecto que tenga tres objetivos. En primer lugar, utilizando la misma metodología empleada en este estudio, determinar qué vocativos reciben los hombres en la Feria del Agricultor. Esto permitiría averiguar si el trato a hombres y a mujeres es diferente, como hallaron Wolfson y Manes (1980) en los Estados Unidos. El segundo objetivo será entrevistar a los vendedores para averiguar qué factores inciden en su escogencia de vocativos. Solamente con un estudio cuidadoso de estos datos se podrá trascender estereotipos y comprender los verdaderos propósitos que determinan el uso o la ausencia de vocativos de estos hablantes. Finalmente, me propongo entrevistar a una muestra aleatoria de compradores y compradoras de la Feria del Agricultor para averiguar cómo perciben ellos los vocativos que reciben de los vendedores. Estas percepciones indudablemente enriquecerán nuestra comprensión de la cortesía verbal en el español de Costa Rica.

\section{Bibliografía}

Arias Núñez, Carolina. 2002. Los tratamientos en el español intermontano central de Costa Rica, área metropolitana: un análisis socio-pragmático. Tesis para optar por el grado de Licenciatura en Filología Española: Universidad de Costa Rica.

Brown, Penelope y Stephen Levinson. 1987. Politeness: Some universals in language use. Cambridge: Cambridge University Press.

Butturff, Douglas y Edmund L. Epstein. 1978. Women's language and style. Akron: University of Akron Press.

Coates, Jennifer. 1998. Language and gender: A reader. Massachusetts: Blackwell Publishers.

Fonseca Zúñiga, Cecilia. 1999. “Sí, mi amor. . .” La Nación, 22 de agosto: 13-A.

Gardner, Carol Brooks. 1980. "Passing by: Street remarks, address rights, and the urban female”. Sociological Inquiry. 50 (3-4): 328-56.

Passing by: Gender and public harassment. Berkeley: University of California Press. 
Henley, Nancy M. 1977. Body politics: Power, sex and non verbal communication. New York: Simon \& Schuster.

Hernández, Annabelle. 1999. "Analysis and comparison of complementing behavior in Costa Rican Spanish and American English”. Revista de Artes y Letras Káñina. 23 (3): 121-31.

Holmes, Janet. 1992. An introduction to sociolinguistics. NY: Longman

1998. "Complimenting: A positive politeness strategy". En: Coates (ed.): 100-20.

Lakoff, Robin. 1975. Language and woman's place. New York: Harper Colophon Books.

McConnell-Ginet, Sally. 1978. "Address forms in sexual politics”. En: Butturff y Epstein (eds.): 23-35.

McConnell-Ginet, Sally, Ruth Borker y Nelly Furman (eds.) 1980. Women and language in literature and society. New York: Praeger.

Murillo Medrano, Jorge. 2003. "La cortesía verbal en situaciones de habla en Costa Rica: hacia la comprensión de la imagen social en su contexto sociocultural". Actas del Primer Coloquio del Programa EDICE "La perspectiva no etnocentrista de la cortesía: identidad sociocultural de las comunidades hispanohablantes.": 128-42. Stockholms Universitet.

Pavez Phillips, Luciana. 2002. Damitas hermosas ¿por qué se esconden? Cortesía lingüística e imagen en las salas de chat. Tesis para optar por el grado de Maestría en Lingüística: Universidad de Costa Rica.

Poynton, Cate. 1989. Language and gender: Making the difference. New York: Oxford University Press.

Wolfson, Nessa y Joan Manes. 1980. "Don't dear me!" En: McConnell-Ginet, Borker y Furman (eds.) Women and language in literature and society: 79-92.

Wolfson, Nessa. 1989. Perspectives: Sociolinguistics and TESOL. Massachusetts: Heinle \& Heinle Publishers. 